\title{
Prediction of navigation by visual aesthetics when presented with binary choices.
}

\author{
Charlotte Croucher \\ University of Portsmouth: dept. of Creative Technology \\ Portsmouth, United Kingdom \\ Charlotte.croucher@port.ac.uk
}

\author{
Andreea Molnar \\ University of Portsmouth: dept. of Creative Technology \\ Portsmouth, United Kingdom \\ Andreea.molnar@port.ac.uk
}

\begin{abstract}
This preliminary study explored the possibility of influencing participant's navigation when presented with 5 binary choices. Each participant experienced one trial of the 5 fixed ordered conditions. A think-aloud protocol and questionnaires were used to understand perception and behaviour. We conclude with suggestions of further research of understanding visual aesthetics and their influence of human movement behaviour on patient's navigation
\end{abstract}

Keywords - navigation; wayfinding; virtual reality; visual aesthetics; prediction of choice

\section{INTRODUCTION}

Virtual rehabilitation often requires patients to explore virtual surroundings, whilst participating in repetitive tasks to achieve therapeutic goals and increase independence (1, 2).

It is important to understand cognitive processes, during navigation (3), but generally navigational studies focus on a patient's recollection of route layouts and landmarks rather than the cognitive processes $(3,4)$, and navigational cues are not thoroughly understood. A reduced amount of cues in an environment may make participants feel lost during redirected walking (5). Therefore it is important to understand reactions to navigation cues and the decision process made.

\section{STUDY DESIGN}

This study investigates the decision making and prediction of navigation, rather than exploring the process of navigation using techniques such as spatial navigation. The study looks at conscious and subconscious behaviour when presented with binary navigational choices in a virtual environment, and to explore the possibility of being able to influence navigational choices, when participants have the ability to choose a preferred route. This study explored participant's perception both during and after the study using a think-aloud protocol followed by questionnaires. The equipment consisted of the Wii-balance board, computer mouse and an oculus rift (fig 1, bottom right). Participants were given written instructions detailing the

\author{
Vaughan Powell \\ University of Portsmouth: dept. of Creative Technology \\ Portsmouth, United Kingdom \\ Vaughan.powell@port.ac.uk \\ Wendy Powell \\ University of Portsmouth: dept. of Creative Technology \\ Portsmouth, United Kingdom \\ Wendy.powell@port.ac.uk
}

study protocol and were familiarised with the controls to ensure they understood.

\section{DEVELOPMENT OF CHOICES}

The choices within the environment were classified as either reassuring (expected choice) or discouraging (unexpected choice). The expected paths at each of the five binary choices are as follows: Left, Right, Right, Right, and Right.

In a previous study, participants had shown a level of interaction with characters in a virtual environment, both positively and negatively, whilst being drawn to a tunnel (6). Therefore choice 1 has a populated 'reassuring' choice and choice 2 has a tunnel 'reassuring' choice. Both choice 3 and 4 are designed to create fear and cause tension. Evoking protection mechanisms against environmental and predatory hazards, often shown in video games through low level lighting to hide enemies (7). Choice 3 uses an open path 'reassuring choice' and choice 4 uses light as a 'reassuring' choice. Choice 5 uses downhill for a 'reassuring' choice, with uphill associated with greater effort. (Fig 1 illustrates the 5 choices)

\section{PARTICIPANTS}

There was a total of 8 participants, 5 male and 3 female. Participant 5 has ADHD and Participant 2 has arthritis. Participant age range was 20-72 (mean 39). Two participants (5 and 8 ) had previous familiarity with virtual reality. Additionally there were five participants $(4,5,6,7$, and 8) who played videogames.

\section{RESULTS AND DISCUSSION}

Participant 2 was anxious about using the mouse because of their arthritis, therefore withdrew from the study. However, although the remaining sample size was small, patterns began to emerge from the data however interpretation will be limited at this stage but suggests further work is appropriate. 
For each choice, 4 out of 5 of the gaming participants, choose the same choice as one another whilst predominantly

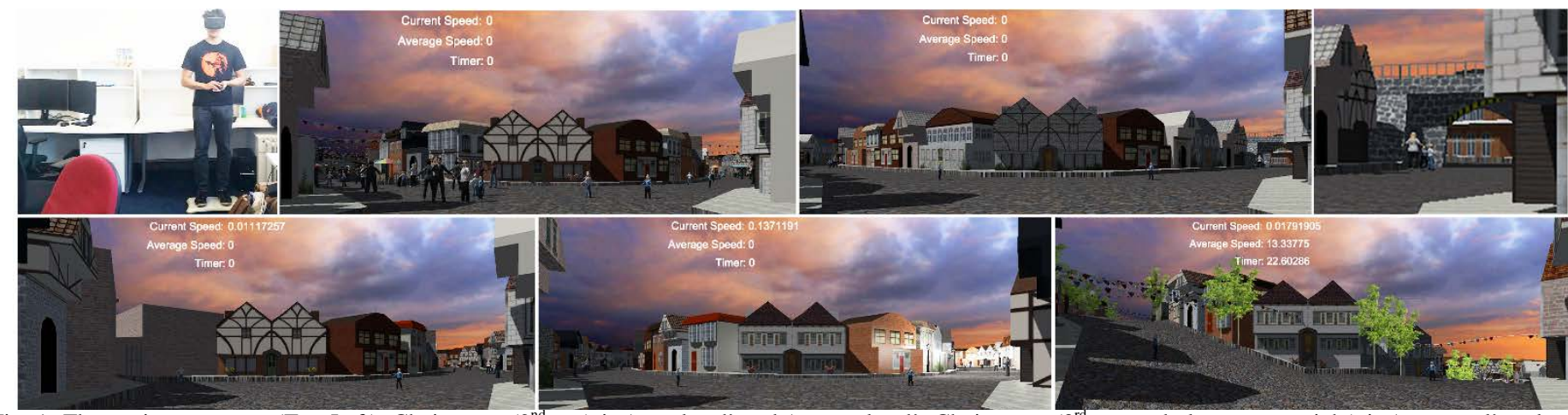

Fig. 1. The equipment setup (Top Left). Choice one ( ${ }^{\text {nd }}$ top) is 'populated' and 'unpopulated'. Choice two ( $3^{\text {rd }}$ top and close up top right) is 'no tunnel' and 'tunnel', Choice three (Bottom Left) is 'alleyway' 'open path'. Choice four (bottom middle), is 'dark' and 'light'. Choice five (bottom right) is 'uphill' and 'downhill'

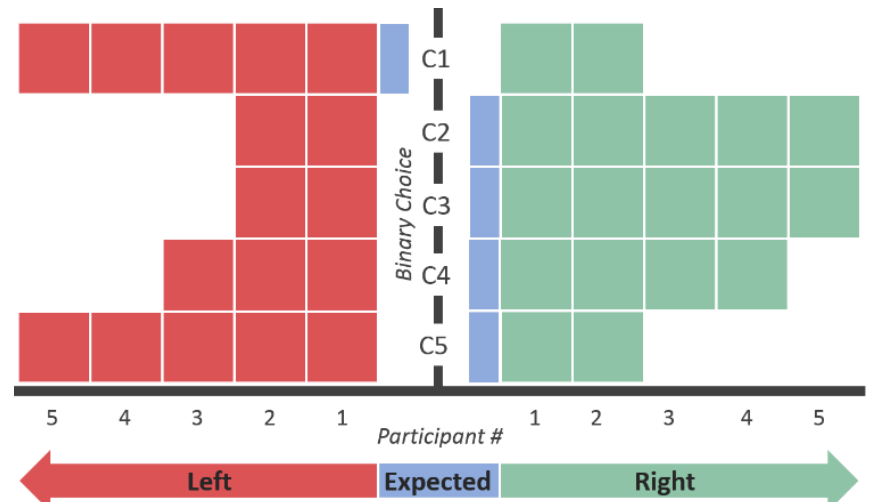

Fig. 2. Participant Choices (key: Red - Left, Blue - Expected choice. Green - Right)

Participant 4 chose the discouraging choice on both choice 3 and 4 , in contrast to the other gaming participants. Both choices used game theory to elicit fear so the discouraging path would not be chosen, therefore this is a counterintuitive finding. However, Participant 4 mentioned their most played game is Gears of War (8), which is a game that encourages exploration through gameplay mechanics. This suggests that participant 4 may have chosen their choices based upon wanting to explore the environment.

Participant 8 desired to travel backwards along the wellstructured paths. This was unexpected, as they knew there would be five choices during the study. Consequently patients may not always wish to follow well-structured paths, possibly restricting patient's achieving therapeutic goals.

\section{CONCLUSION}

In spite of the small size and heterogeneity of the sample, patterns began to emerge when predicting navigation. Some participants made counterintuitive decisions. Underlying gameplay behaviour appeared to affect their decisions, with exploratory behaviour more apparent in the 5 participants who play video games.

This preliminary study indicates that navigational choices may be predicted by visual design, but it is important to consider that prior experience such as with certain game play mechanics may result in individual differences. Additionally patients may not wish to follow well-structured paths, which may make them feel constrained and possibly respond negatively.

If the design of virtual rehabilitation applications are to be better understood, in order to influence behaviour it is important to further explore different design elements and their effect on navigation decision. For example presenting the conditions in other orders and whether they appear to participants on the left or right.

\section{REFERENCES}

[1] J. Halton. "Virtual rehabilitation with video games: a new frontier for occupational therapy.” Occupational Therapy Now, 2008 9.6: 12-14

[2] T.P. Yamato, J.E. Pompeu, S.M.A.A. Pompeu, L. Hassett "Virtual reality for stroke rehabilitation.” Physical Therapy 2016;96(10):1508-13. PubMed PMID:118521016

[3] M. H. G. Claessen, I. J.M. Van der ham, J. M. A. Visser-Meily, E. Jagersma. "Navigation strategy training using virtual reality in six chronic stroke patients: A novel and explorative approach to the rehabilitation of navigation impairment."

Neuropsychological Rehabilitation. 2015 Jun 5:1-25. 2016

[4] I. J. Van der Ham, A. M. Faber, M. Venselarr, M. J. van Kreveld, M. Loffler. "Ecological validity of virtual environments to assess human navigation ability.” Frontiers in psychology. 2015, 6.

[5] K. Vasylevska, H. Kaufmann, M. Bolas, E. A. Suma. "Flexible spaces: dynamic layout generation for infinite walking in virtual environments." In 3D user interfaces (3DUI), 2013 IEEE Symposium on 2013 Mar 16 (pp. 39-42). IEEE

[6] C. Croucher, V. Powell, A. Molnar, W. Powell. "Visual elements influence on navigation in virtual environments.” In $11^{\text {th }}$ international conference on disability, virtual reality and associated technologies, 287-290. Los Angeles: Sharkey, P., Rizzo, A. A., 2016. 
C. W. Totten. “An architectural approach to level design.” CRC Press, 2014

[8] Games, Epic. "Gears of War” [computer game]. Washington
US:
Microsoft
Games
Studios.
2006 
